\title{
Review of: "Strength criterion of rock mass considering the damage and effect of joint dip angle"
}

\author{
Zhenyue $\mathrm{Shi}^{1}$ \\ 1 Shandong University of Science and Technology
}

Potential competing interests: The author(s) declared that no potential competing interests exist.

The detailed consideration of the damage model in this paper is worth studying and sharing, especially the initial damage problem.

At the same time, the author verifies the model, which is closer to the practice, so it can be seen that the theoretical research has applicability. 